\title{
Bayesian sandwich posteriors for pseudo-true parameters
}

\author{
Peter Hoff and Jon Wakefield \\ Departments of Statistics and Biostatistics \\ University of Washington
}

June 14, 2021

\begin{abstract}
Under model misspecification, the MLE generally converges to the pseudo-true parameter, the parameter corresponding to the distribution within the model that is closest to the distribution from which the data are sampled. In many problems, the pseudo-true parameter corresponds to a population parameter of interest, and so a misspecified model can provide consistent estimation for this parameter. Furthermore, the well-known sandwich variance formula of Huber (1967) provides an asymptotically accurate sampling distribution for the MLE, even under model misspecification. However, confidence intervals based on a sandwich variance estimate may behave poorly for low sample sizes, partly due to the use of a plug-in estimate of the variance. From a Bayesian perspective, plug-in estimates of nuisance parameters generally underrepresent uncertainty in the unknown parameters, and averaging over such parameters is expected to give better performance. With this in mind, we present a Bayesian sandwich posterior distribution, whose likelihood is based on the sandwich sampling distribution of the MLE. This Bayesian approach allows for the incorporation of prior information about the parameter of interest, averages over uncertainty in the nuisance parameter and is asymptotically robust to model misspecification. In a small simulation study on estimating a regression parameter under heteroscedasticity, the addition of accurate prior information and the averaging over the nuisance parameter are both seen to improve the accuracy and calibration of confidence intervals for the parameter of interest.
\end{abstract}

Keywords: estimating equations, exponential family, model misspecification, pivotal quantity.

This note is part of a discussion of "Bayesian inference with misspecified models" by Stephen Walker. Replication code for the simulation study is available at the first author's website: www.stat.washington.edu/ hoff. 


\section{Introduction}

Let $X$ be the data resulting from an experiment, survey or random process, and let $\theta$ denote some fixed but unknown aspect of the the data generating process. Before the experiment is run, both $X$ and $\theta$ are uncertain. A subjective Bayesian uses probability to represent pre-experimental uncertainty in both $X$ and $\theta$, and Bayes' rule to represent uncertainty in $\theta$ after having observed $X$. One appealing aspect of the subjective Bayesian approach is that it is an internally consistent and rational way to update information. If $\mathcal{P}_{\Theta}=\{p(X \mid \theta): \theta \in \Theta\}$ expresses our beliefs about $X$ given $\theta$, and $\pi(\theta)$ expresses our beliefs about $\theta$, then $\pi(\theta \mid X) \propto \pi(\theta) p(X \mid \theta)$ expresses what we should believe about $\theta$, having observed $X$. For $\pi(\theta \mid X)$ to be of most use, both $p(X \mid \theta)$ and $\pi(\theta)$ should actually represent our beliefs, at least approximately. Professor Walker's paper (Walker, 2013) highlights the problem that in practice, a statistical model $\mathcal{P}_{\Theta}$ is often used that is known to not represent beliefs, in that it is suspected that $\mathcal{P}_{\Theta}$ does not include the distribution that generated the data. In such cases, interpretation of $\pi(\theta \mid X)$ may be problematic: Not only is the validity of $\pi(\theta \mid X)$ as a probabilistic description of information about $\theta$ potentially invalid, it is not even clear that $\theta$ represents anything of interest.

One remedy discussed by Walker is to expand the model so that $\mathcal{P}_{\Theta}$ can be assumed to contain the correct data generating process, or at least something very close to it. Depending on what the data are, this can make the model quite large. Walker focuses on the situation where the data are taken to be a sample of observations from a population, i.e. $X=\left\{x_{1}, \ldots, x_{n}\right\}$. To guarantee that the model is not misspecified, $\mathcal{P}_{\Theta}$ must be quite large, essentially covering (in a topological sense) the space of all probability distributions. However, addressing the model misspecification problem in this way can complicate the other component of subjective Bayesian inference - specification of the prior distribution. The larger the model is, the more difficult it will be to specify a prior that represents actual beliefs about the unknown population. If $\pi(\theta)$ does not represent prior beliefs, then the use of $\pi(\theta \mid X)$ as an expression of posterior beliefs is questionable, except possibly when the sample size is very large.

\section{Incorrect models with correct pseudo-true parameters}

If we wish to benefit from the internal consistency of subjective Bayesian inference, we need to limit our probability statements to those quantities about which we have actual information. As a very simple example, suppose we have a sample $x_{1}, \ldots, x_{n}$ of independent measurements for which the measurement error $\sigma^{2}$ is known. If we have prior information $\pi(\theta)$ about the population mean $\theta$, but not any other aspect of the population (other than $\sigma^{2}$ ), then we should limit our data $X$ to quantities whose sampling distribution depends only on $\theta$ and $\sigma^{2}$. This condition will be approximately met by the sample mean $\bar{x}$, whose sampling distribution is approximately normal. A limited form of 
subjective Bayesian inference can proceed via the posterior density $\pi(\theta \mid \bar{x}) \propto \pi(\theta) \times p(\bar{x} \mid \theta)$, where the latter density is that of a $N\left(\theta, \sigma^{2} / n\right)$ random variable.

Strictly speaking, the model $p(\bar{x} \mid \theta)$ is misspecified unless $x_{1}, \ldots, x_{n}$ are sampled from a normal population. As Walker (2013) asks, what does $\theta$ represent in the case of model misspecification? Letting $p_{0}(\bar{x})$ be the true sampling distribution of $\bar{x}$, the pseudo-true parameter $\theta^{*}$ is given by

$$
\begin{aligned}
\theta^{*} & =\arg \min _{\theta} \int \log \frac{p_{0}(\bar{x})}{p(\bar{x} \mid \theta)} p_{0}(\bar{x}) d \bar{x} \\
& =\arg \min _{\theta} \int \frac{1}{2}\left[\log \left(2 \pi \sigma^{2} / n\right)+n \bar{x}^{2} / \sigma^{2}-2 n \bar{x} \theta / \sigma^{2}+n \theta^{2} / \sigma^{2}\right] p_{0}(\bar{x}) d \bar{x} \\
& =\arg \min _{\theta}\left(\theta^{2}-2 \theta \mathrm{E}[\bar{x}]\right)=\mathrm{E}[\bar{x}]=\theta,
\end{aligned}
$$

and so in this case, the pseudo-true parameter is equal to the parameter of interest, regardless of whether or not $p_{0}$ is in the model. Furthermore, the posterior distribution given by $\pi(\theta \mid \bar{x}) \propto$ $\pi(\theta) \times p(\bar{x} \mid \theta)$ provides (approximate) subjective Bayesian inference for the population mean $\theta$, even if the population is not normal, and without having to quantify prior information about anything but the first two population moments.

Now suppose we are interested in estimating a collection of population moments $\boldsymbol{\lambda} \in \mathbb{R}^{p}$, where $\lambda_{j}=\mathrm{E}\left[g_{j}(x)\right], j=1, \ldots, p$. Is there a parametric model $\{p(x \mid \theta): \theta \in \Theta\}$ whose pseudo-true parameter $\theta^{*}$ satisfies $\mathrm{E}\left[g_{j}(x) \mid \theta^{*}\right]=\lambda_{j}$ for each $j=1, \ldots, p$ ? Consider the exponential family with sufficient statistics $\left\{g_{1}(x), \ldots, g_{p}(x)\right\}$ given by $p(x \mid \theta)=h(x) \exp \left\{\theta_{1} g_{1}(x)+\cdots \theta_{p} g_{p}(x)-c(\theta)\right\}$. The pseudo-true parameter $\theta^{*}$ for such a model is given by

$$
\begin{aligned}
\theta^{*} & =\arg \min _{\theta} \int \log \frac{p_{0}(x)}{p(x \mid \theta)} p_{0}(x) d x \\
& =\arg \max _{\theta} \int[\log p(x \mid \theta)] p_{0}(x) d x \\
& =\arg \max _{\theta} \int\left[\theta_{1} g_{1}(x)+\cdots \theta_{p} g_{p}(x)\right] p_{0}(x) d x-c(\theta),
\end{aligned}
$$

where $p_{0}(x)$ is the true population density. Taking derivatives with respect to each element of $\theta$ tells us that $\theta^{*}$ is the solution in $\theta$ to

$$
\frac{\partial}{\partial \theta_{j}} \int\left[\theta_{1} g_{1}(x)+\cdots \theta_{p} g_{p}(x)\right] p_{0}(x) d x=\frac{\partial}{\partial \theta_{j}} c(\theta) .
$$

The left-hand side is $\int g_{j}(x) p_{0}(x) d x=\lambda_{j}$, one of the moments we want to estimate. The righthand side is equal to $\mathrm{E}\left[g_{j}(x) \mid \theta\right]$, due to the well-known identity for exponential families. Therefore, $\theta^{*}$ is the parameter value such that $\int g_{j}(x) p\left(x \mid \theta^{*}\right) d x=\int g_{j}(x) p_{0}(x) d x$ for $j \in\{1, \ldots, p\}$. Thus for an exponential family with sufficient statistic $\left\{g_{1}(x), \ldots, g_{p}(x)\right\}$, the pseudo-true parameter $\theta^{*}$ satisfies $\mathrm{E}\left[g_{j}(x) \mid \theta^{*}\right]=\mathrm{E}\left[g_{j}(x)\right]$, where the latter expectation is with respect to the true population distribution. 
The result above suggests that some models can be used to make inference for certain aspects of a population $P_{0}$, even if $P_{0}$ is not a member of the model. Specifically, a possibly incorrect model $\left\{P_{\theta}: \theta \in \Theta\right\}$ can be used to obtain consistent estimators of those functionals of $P_{0}$ which match those of $P_{\theta^{*}}$, where $\theta^{*}$ is the pseudo-true parameter. However, this does not ensure that the model can correctly represent the sampling variability of such estimators, even asymptotically. As a result, confidence intervals based on an incorrect model can be asymptotically invalid, even if the incorrect model provides a consistent estimator. To address this concern, Huber (1967) derived the limiting distribution of the MLE $\hat{\theta}$ of $\theta$ under a possibly incorrect model in terms of the pseudotrue parameter. The approximation proceeds roughly as follows: Suppose $x_{1}, \ldots, x_{n}$ are i.i.d. observations from population $P_{0}$, and let $l\left(\theta: x_{i}\right)=\log p\left(x_{i} \mid \theta\right)$ be the log-likelihood corresponding to a single observation $x_{i}$. A first order Taylor series expansion of $\sum \dot{i}\left(\theta^{*}: x_{i}\right)$ around the MLE $\hat{\theta}$ gives

$$
\sum_{i=1}^{n} \dot{l}\left(\theta^{*}: x_{i}\right) \approx\left(\sum_{i=1}^{n} \ddot{l}\left(\hat{\theta}: x_{i}\right)\right)\left(\theta^{*}-\hat{\theta}\right) .
$$

By the central limit theorem, the sum on the left-hand side is approximately $N(0, n B)$, where $B=\operatorname{Var}\left[\dot{l}\left(\theta^{*}: x\right)\right]$ and the variance here is under $P_{0}$. Letting $A=\sum_{i=1}^{n} \ddot{l}\left(\hat{\theta}: x_{i}\right)$ be the sum on the right-hand side, we have

$$
\left(\theta^{*}-\hat{\theta}\right) \dot{\sim} N\left(0, n A^{-1} B A^{-1}\right),
$$

where "ن்" means "approximately distributed as." This result has been used extensively to obtain confidence intervals for the pseudo-true parameter $\theta^{*}$, in cases where it corresponds to a population quantity of interest. In practice, since $\theta^{*}$ is unknown, $B$ is estimated as $\hat{B}=\sum \dot{l}\left(\hat{\theta}: x_{i}\right) \dot{l}\left(\hat{\theta}: x_{i}\right)^{T} / n$, the sample variance of the likelihood functions at the MLE. The resulting variance estimate $\hat{C}=$ $n A^{-1} \hat{B} A^{-1}$ is called the sandwich variance estimate for $\left(\theta^{*}-\hat{\theta}\right)$. Confidence intervals for $\theta^{*}$ can be obtained by approximating the distribution of $\hat{C}^{-1 / 2}\left(\theta^{*}-\hat{\theta}\right)$ by a $N(0, I)$ distribution. Sandwich confidence intervals avoid the issue of model misspecification by positing the sampling distribution of the pivotal quantity $\hat{C}^{-1 / 2}\left(\theta^{*}-\hat{\theta}\right)$, rather than the sampling distribution of $x_{1}, \ldots, x_{n}$. The model used to obtain the likelihoods $\left\{l\left(\theta: x_{i}\right), i=1, \ldots, n\right\}$ is simply a tool that provides a consistent estimate of the pseudo-true parameter $\theta^{*}$ and asymptotically correct confidence intervals via the sandwich variance estimate. A review of the theory and methods for sandwich-based data analysis appears in White (1982), and several applications are described in White (1980) and Rovall (1986). Sandwich variance estimation has also been applied to inference based on generalized estimating equations (GEE), a popular likelihood-free approach to inference (Liang and Zeger, 1986; Zeger and Liang, 1986; Gourieroux et al., 1984). 


\section{A Bayesian sandwich posterior distribution}

While used extensively in practice, sandwich confidence intervals can behave poorly for low sample sizes, with coverage often being well below their nominal level (Kauermann and Carroll, 2001). One reason for this is that the sandwich procedure does not properly account for uncertainty in the variance $B$ of $\dot{l}\left(\theta^{*}: x\right)$. The replacement of $B$ by $\hat{B}$ in fact uses two plug-in approximations: the MLE $\hat{\theta}$ for $\theta^{*}$, and the sample covariance $\hat{B}$ for the population covariance $B$. Ignoring the uncertainty in both of these approximations is likely to provide an underestimate of $B$, resulting in overly-narrow confidence intervals and below-nominal coverage rates.

One of the attractions of Bayesian inference is that uncertainty in nuisance parameters can be accounted for by integrating over their possible values, rather than plugging in point estimates. With this in mind, we propose the following version of a "Bayesian sandwich" posterior distribution, quantifying the uncertainty in both $\theta^{*}$ and $B$ : Given a working model $\mathcal{P}_{\Theta}=\{p(x \mid \theta): \theta \in \Theta\}$ and observations $x_{1}, \ldots, x_{n} \sim$ i.i.d. $P_{0}$, we form a likelihood derived from the approximate joint density of the MLE $\hat{\theta}$ based on $\mathcal{P}_{\Theta}$, and the sum of squares of the derivatives of the log-likelihood functions $S(\theta)=\sum_{i=1}^{n} \dot{l}\left(\theta: x_{i}\right) \dot{l}\left(\theta: x_{i}\right)^{T}$, giving us the following approximate likelihood function:

$$
\begin{aligned}
p\left(\hat{\theta}, S\left(\theta^{*}\right) \mid \theta^{*}, B\right) & =p\left(\hat{\theta} \mid \theta^{*}, B\right) \times p\left(S\left(\theta^{*}\right) \mid \hat{\theta}, \theta^{*}, B\right) \\
& \approx \operatorname{dnorm}\left(\hat{\theta} \mid \theta^{*}, n A^{-1} B A^{-1}\right) \times \operatorname{dWishart}\left(S\left(\theta^{*}\right) \mid n, B\right),
\end{aligned}
$$

where "dnorm" and "dWishart" refer to the normal and Wishart densities respectively. The validity of this likelihood is based on three approximations. The first is the normal approximation to the distribution of $\hat{\theta}$ given by (11). The second is the conditional independence of $S\left(\theta^{*}\right)$ and $\hat{\theta}$ and the third is the approximation of the distribution of $S\left(\theta^{*}\right)$ with a Wishart distribution. The first of these approximations is justified asymptotically by Huber (1967), whereas the latter two are, at least currently, heuristic.

Based on this approximate likelihood and a prior distribution for $\left(\theta^{*}, B\right)$, a posterior distribution can be obtained via MCMC in the usual way. For example, if the priors for $\theta^{*}$ and $B$ are normal $\left(m_{0}, V_{0}\right)$ and inverse-Wishart $\left(\nu_{0}, S_{0}^{-1}\right)$ respectively, then posterior approximation can proceed via the following Gibbs sampler: Given current values of $\theta_{(s)}^{*}$ and $B_{(s)}$,

1. simulate $\theta_{(s+1)}^{*} \sim N_{p}\left(m_{1}, V_{1}\right)$, where

$$
V_{1}^{-1}=V_{0}^{-1}+A B_{(s)}^{-1} A / n, m_{1}=V_{1}\left[V_{0}^{-1} \theta_{0}+A B_{(s)}^{-1} A \hat{\theta} / n\right]
$$

2. simulate $B_{(s+1)}^{-1} \sim \operatorname{Wishart}\left(\nu_{1}, S_{1}^{-1}\right)$, where $\nu_{1}=\nu_{0}+n+1$ and

$$
S_{1}=S_{0}+S\left(\theta_{(s+1)}^{*}\right)+A\left(\theta_{(s+1)}^{*}-\hat{\theta}\right)\left(\theta_{(s+1)}^{*}-\hat{\theta}\right)^{T} A / n .
$$


The hyperparameters $\left(m_{0}, V_{0}\right)$ should ideally represent prior information about $\theta^{*}$. Information upon which to base $\left(\nu_{0}, S_{0}^{-1}\right)$ might be harder to come by. One possibility would be to use Jeffreys' prior, $\pi(B) \propto|B|^{-(p+1) / 2}$ (Geisser and Cornfield, 1963). The posterior distribution under this prior can be approximated with the above Gibbs sampler by setting $\nu_{0}=0$ and $S_{0}$ equal to the $p \times p$ matrix of zeros.

\section{Example: Regression with heteroscedastic errors}

Suppose we have a sample $\left(y_{1}, x_{1}\right), \ldots,\left(y_{n}, x_{n}\right) \sim$ i.i.d. $P_{0}$, and wish to estimate the linear regression of $y$ on $x$ that would be obtained from performing the regression on the entire population. In other words, letting $\boldsymbol{x}=(1, x)$, we want to estimate $\boldsymbol{\beta}=\mathrm{E}\left[\boldsymbol{x} \boldsymbol{x}^{T}\right]^{-1} \mathrm{E}[\boldsymbol{x} y]$, where both expectations are under $P_{0}$. Consistent estimation of this quantity can be obtained from the normal regression model $y_{i}=\boldsymbol{\beta}^{T} \boldsymbol{x}_{i}+\epsilon_{i}, \epsilon_{1}, \ldots, \epsilon_{n} \sim$ i.i.d. $N(0,1)$, even if $P_{0}$ is not in this model, as the pseudo-true parameter of this regression model is equal to $\mathrm{E}\left[\boldsymbol{x} \boldsymbol{x}^{T}\right]^{-1} \mathrm{E}[\boldsymbol{x} y]$, the population regression parameter under $P_{0}$. We also note that the variance of the error terms in the regression model can be taken to be any fixed value: Whichever value is specified will end up canceling out in the sandwich variance calculation.

Using the regression model as our working model, we have $i(\boldsymbol{\beta}: y, \boldsymbol{x})=\boldsymbol{x}\left(y-\boldsymbol{\beta}^{T} \boldsymbol{x}\right)$ and $\ddot{l}(\boldsymbol{\beta}: y, \boldsymbol{x})=-\boldsymbol{x} \boldsymbol{x}^{T}$, giving

$$
A=-\sum_{i=1}^{n} \boldsymbol{x}_{i} \boldsymbol{x}_{i}^{T} \text { and } S(\boldsymbol{\beta})=\sum_{i=1}^{n} \boldsymbol{x}_{i}\left(y_{i}-\boldsymbol{\beta}^{T} \boldsymbol{x}_{i}\right)
$$

The usual sandwich variance estimate of the MLE $\hat{\boldsymbol{\beta}}$ under the normal regression model is $n A^{-1} \hat{B} A^{-1}$ where $\hat{B}=S(\hat{\boldsymbol{\beta}}) / n$. In contrast, the proposed Bayesian sandwich posterior distribution infers $B$ jointly with $\boldsymbol{\beta}$, based on the Wishart model for $S(\boldsymbol{\beta})$. To compare the performance of the proposed Bayesian sandwich posterior to the usual sandwich procedure, we ran a small simulation study in order to calculate coverage rates and average interval widths of nominal $95 \%$ confidence intervals. For both small $(n=10)$ and large $(n=500)$ sample sizes, datasets were generated as $x_{1}, \ldots, x_{n} \sim$ i.i.d. exponential(1), and $y_{i} \mid x_{i} \sim N\left(\beta_{1}+\beta_{2} x_{i},\left(\beta_{1}+\beta_{2} x_{i}\right)^{2}\right)$, where $\beta_{1}=\beta_{2}=1$. Thus the working model incorrectly assumes homoscedastic errors, whereas the true population has substantial heteroscedasticity.

For each simulated dataset, we obtained Bayesian sandwich posterior distributions under four different priors of the form $\pi(\boldsymbol{\beta}, B)=\pi(\boldsymbol{\beta}) \pi(B)$, based on two choices for each of $\pi(\boldsymbol{\beta})$ and $\pi(B)$. The priors for $\boldsymbol{\beta}$ included the (improper) uniform prior on $\mathbb{R}^{2}$, and an informative $N\left((1,1)^{T}, n A^{-1}\right)$ prior distribution. This latter prior, weakly centered around the correct values, represents accurate but weak information about $\boldsymbol{\beta}$ that someone may have: The matrix $A=\sum \boldsymbol{x}_{i} \boldsymbol{x}_{i}^{T}$ is the information for $\boldsymbol{\beta}$ from $n$ observations, and so $A / n$ represents the information equivalent of one observation. 


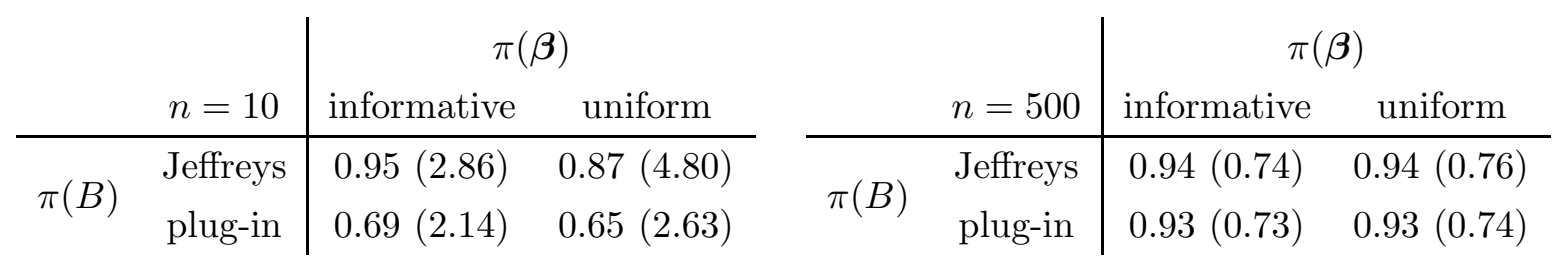

Table 1: Coverage rates and average interval widths (in parentheses) of 10,000 nominal 95\% confidence intervals based on the four procedures. The standard (non-Bayesian) sandwich procedure corresponds closely to the uniform/plug-in prior combination.

The priors for $B$ included Jeffreys' prior and a point-mass prior on $\hat{B}$, the plug-in estimate of $B$. We note that the uniform/plug-in combination of priors leads to a $N_{p}\left(\hat{\boldsymbol{\beta}}, n A^{-1} \hat{B} A^{-1}\right)$ posterior distribution for $\boldsymbol{\beta}$. This posterior was referred to as the "artificial posterior" by Müller (2011), who compared the risk of the resulting estimator to the risk of the Bayes estimator from the working model.

For each sample size we simulated 10,000 datasets from the heteroscedastic regression distribution given above, and obtained $95 \%$ posterior confidence intervals for the slope $\beta_{2}$ based on each of the four priors. Empirical coverage probabilities and average interval widths are given in Table 1. For each dataset we also obtained a Wald-type interval for $\beta_{2}$ based on the plug-in sandwich variance estimate (the usual sandwich confidence interval), but it performed nearly identically to the estimator based on the uniform/plug-in prior, so we do not report these results separately.

For $n=10$, both plug-in procedures perform very poorly in terms of coverage. This seems primarily due to underestimation of $B$, resulting in confidence intervals that are shorter than are required to attain $95 \%$ coverage. In contrast, the procedures using Jeffreys' prior both take uncertainty in $B$ into account, and provide coverage rates closer to the nominal value. However, the absence of any prior information about $\boldsymbol{\beta}$ (uniform $\pi(\boldsymbol{\beta})$ ) leads to interval widths that are quite high as compared to those obtained with some prior information (informative $\pi(\boldsymbol{\beta})$ ), as we would expect: Accurate prior information about $\boldsymbol{\beta}$ leads to more precise inference. For $n=500$, all sandwich-based procedures performed similarly, reflecting the asymptotic correctness of sandwichbased confidence intervals in general. This is in contrast to the $95 \%$ nominal posterior confidence intervals based on the (uncorrected) misspecified regression model. For a sample size of $n=500$ and under the informative prior described above, these $95 \%$ posterior confidence intervals had a coverage rate of only $68 \%$. 


\section{Discussion}

Bayesian inference typically proceeds via the formulation of a sampling model for the data $X$ and a prior distribution over the sampling model. To guard against model misspecification, one approach is to make the model large enough to ensure that it contains the distribution that generated the data. However, such a large model can lead to difficulties in prior specification and posterior calculation. Such difficulties can often be avoided when interest is limited to a simple low-dimensional parameter $\theta$. In such cases there often exists a statistic $t(X)$ or pivotal quantity $s(X, \theta)$ whose sampling distributions are robust to model misspecification and from which a likelihood can be constructed. In this note, we have suggested using the asymptotic "sandwich" distribution of the MLE to construct a likelihood, and have illustrated via simulation how Bayesian confidence intervals based on this likelihood provide improved performance over the standard non-Bayesian procedure. Other authors have used similar ideas previously: In a testing context, Johnson (2005) shows how modeling the distribution of test statistics, rather than the individual observations, can lead to great simplifications in the calculation of Bayes factors (see also Wakefield (2009)). In a semiparametric estimation setting, Hoff (2007) proposes Bayesian inference via a marginal likelihood that depends only on the parameter of interest and not an infinite-dimensional nuisance parameter. Approaches such as these suggest that simple, robust Bayesian inference can be obtained by restricting attention to only those aspects of the data for which confident probability statements can be made.

\section{References}

Geisser, S. and J. Cornfield (1963). Posterior distributions for multivariate normal parameters. $J$. Roy. Statist. Soc. Ser. B 25, 368-376.

Gourieroux, C., A. Montfort, and A. Trognon (1984). Pseudo-maximum likelihood methods: theory. Econometrica 52, 681-700.

Hoff, P. D. (2007). Extending the rank likelihood for semiparametric copula estimation. Ann. Appl. Stat. 1(1), 265-283.

Huber, P. (1967). The behavior of maximum likelihood estimators under non-standard conditions. In L. LeCam and J. Neyman (Eds.), Proceedings of the Fifth Berkeley Symposium on Mathematical Statistics and Probability, pp. 221-233. University of California Press.

Johnson, V. (2005). Bayes factors based on test statistics. Journal of the Royal Statistical Society, Series B 67, 689-701.

Kauermann, G. and R. Carroll (2001). A note on the efficiency of sandwich covariance matrix estimation. Journal of the American Statistical Association 96, 1387-1396. 
Liang, K. and S. Zeger (1986). Longitudinal data analysis using generalized linear models. Biometrika 73, 13-22.

Müller, U. (2011). Risk of bayesian inference in misspecified models, and the sandwich covariance matrix. Unpublished Manuscript, Princeton University.

Royall, R. (1986). Model robust confidence intervals using maximum likelihood estimators. International Statistical Review 54, 221-226.

Wakefield, J. (2009). Bayes factors for genome-wide association studies: comparison with p-values. Genetic epidemiology 33(1), 79-86.

Walker, S. G. (2013). Bayesian inference with misspecified models. Journal of Statistical Planning and Inference. to appear.

White, H. (1980). A heteroskedasticity-consistent covariance matrix estimator and a direct test for heteroskedasticity. Econometrica 48, 1721-746.

White, H. (1982). Maximum likelihood estimation of misspecified models. Econometrica 50(1), $1-25$.

Zeger, S. and K. Liang (1986). Longitudinal data analysis for discrete and continuous outcomes. Biometrics 42, 121-130. 